Abstract S55 Table 1 Patient characteristics, EMG para\%max, measures of pulmonary function and exercise performance in thirty patients with CF

\begin{tabular}{|c|c|c|}
\hline & Median & Range \\
\hline Age & 24 & 16 to 47 \\
\hline BMI & 20.6 & 18.0 to 29.4 \\
\hline EMGpara\% max & 7.8 & 2.0 to 34.5 \\
\hline $\mathrm{FEV}_{1}$ (\% pred.) & 63.3 & 16.0 to 101.3 \\
\hline RV/TLC (\%) & 40.6 & 21.5 to 74.0 \\
\hline $\mathrm{FRC}_{\text {pleth }} / \mathrm{TLC}(\%)$ & 58.9 & 41.2 to 78.6 \\
\hline ISWT (M) & 630 & 280 to 880 \\
\hline $\mathrm{VO}_{2 \text { peak }}(\mathrm{ml} / \mathrm{min} / \mathrm{kg})$ & 24.9 & 17.1 to 39.9 \\
\hline
\end{tabular}

plethysmography and an incremental shuttle walking test (ISWT) with breath by breath metabolic data were also performed.

Results Patient characteristics, EMGpara\%max, measures of pulmonary function and exercise performance are shown in Table 1. EMGpara\%max was significantly associated with residual volume/total lung capacity ratio (RV/TLC, $\mathrm{r}=0.724, \mathrm{p}<0.001$ ), forced expiratory volume in 1 second $\left(\mathrm{FEV}_{1} \%\right.$ pred. $\left.\mathrm{r}=-0.648, \mathrm{p}<0.001\right)$ and functional residual capacity/total lung capacity ratio $\left(\mathrm{FRC}_{\text {pleth }} / \mathrm{TLC}, \mathrm{r}\right.$ $=0.625, \mathrm{p}<0.001)$. EMGpara\%max showed the strongest relationship with ISWT distance $(\mathrm{r}=-0.612, \mathrm{p}<0.001)$ and peak oxygen uptake $\left(\mathrm{VO}_{2 \text { peak, }} \mathrm{r}=-0.665, \mathrm{p}=0.001\right)$. Weaker relationships were observed between ISWT distance and pulmonary function $\left(\mathrm{FEV}_{1} \mathrm{r}=\right.$ $0.518 \mathrm{p}=0.006$, RV/TLC $\mathrm{r}=-0.451 \mathrm{p}=0.024, \mathrm{FRC}_{\text {pleth }} / \mathrm{TLC} \mathrm{r}=$ $-0.299 \mathrm{p} 0.147)$ and $\mathrm{VO}_{2 \text { peak }}\left(\mathrm{FEV}_{1} \mathrm{r}=0.521 \mathrm{p}=0.008, \mathrm{RV} / \mathrm{TLC}\right.$ $\mathrm{r}=-0.505 \mathrm{p}=0.014, \mathrm{FRC}_{\text {pleth }} / \mathrm{TLC} \mathrm{r}=-0.389 \mathrm{p}=0.066$ ).

Conclusion EMGpara correlates strongly with conventional pulmonary function measures in $\mathrm{CF}$ and has a closer relationship with exercise capacity than standard pulmonary function parameters. EMGpara\%max therefore represents a promising marker of CF lung disease severity.

\section{S56 DIFFERENCES IN FORCED OSCILLATION TECHNIQUE BETWEEN HEALTHY INDIVIDUALS, OBSTRUCTIVE SLEEP APNOEA AND OBESITY HYPOVENTILATION SYNDROME}

${ }^{1} S$ Mandal, ${ }^{1}$ A Vaughan-France, ${ }^{2} \mathrm{~T}$ Dhir, ${ }^{1} \mathrm{ES}$ Suh, ${ }^{3} \mathrm{P}$ Pompilio, ${ }^{3} \mathrm{R}$ Dellaca, ${ }^{1} \mathrm{~N}$ Hart. ${ }^{1}$ Lane Fox Respiratory Unit, Guy's and St Thomas' Hospital, London, UK; ${ }^{2}$ King's College London, London, UK; ${ }^{3}$ Department of Electronic Informatics and Bioengineering, Politecnico Di Milano, Milan, Italy

\subsection{6/thoraxjnl-2014-206260.62}

Introduction Forced oscillation technique has been used to demonstrate expiratory flow limitation (EFL, by measurement of $\mathrm{DX}_{\mathrm{rs}, 5 \mathrm{~Hz}}$ ) in chronic obstructive pulmonary disease, however, this technique has not been widely used in the obese population. Obese individuals breathe at lower lung volumes and are therefore likely to develop EFL. We have previously demonstrated EFL occurs in individuals with obesity hypoventilation syndrome (OHS) but wished to determine if this also occurred in those with obstructive sleep apnoea (OSA) and compare differences between these groups.

Method Subjects with established OSA, OHS and healthy volunteers were recruited from the Lane Fox Respiratory Unit and Sleep Disorders Centre, St Thomas' Hospital. Subjects underwent measurements of height, weight, spirometry and EFL (ResmonPro, ResTech, Milan, Italy).

Results Eleven healthy (HC), 8 OSA and 9 OHS subjects were recruited, age $23.6 \pm 4.2,31.4 \pm 8.0$ and $58.9 \pm 10.4$ years respectively. Body mass index (BMI): healthy subjects $17.9 \pm 2.9$; OSA group $41.4 \pm 8.0$; OHS group; $46.8 \pm 9.3 \mathrm{~kg} / \mathrm{m}^{2}$, there were significant differences in BMI between the HC and OSA and OHS groups $(\mathrm{p}<0.001)$ but no difference between OSA and OHS. Spirometry (FEV1, FVC): HC $3.54 \pm 1.15,4.35 \pm 1.47$, OSA $2.55 \pm$ $0.85,3.27 \pm 1.03$ OHS $2.04 \pm 0.74,2.58 \pm 0.85$. In both the OSA and OHS groups $\mathrm{DX}_{\mathrm{rs}}$ increased with recumbency, as did the percentage of flow limited breaths (Table 1). Each group significantly increased their inspiratory resistance with the supine position compared to the upright seated position. There was a significant difference in $\mathrm{DX}_{\mathrm{rs}}$ between $\mathrm{HC}$ and OHS only in upright, $45^{\circ}$ and supine positions $(p<0.05)$. There was also a difference in the percentage of EFL breaths between $\mathrm{HC}$ and $\mathrm{OHS}$ in the $45^{\circ}$ and supine positions and between OSA and OHS in the $45^{\circ}$ position $(\mathrm{p}<0.05)$. Conclusion Patients with obesity and sleep disordered breathing experience EFL, which was more evident in the OHS group compared to the OSA group. This may be a consequence of their higher BMI impacting their lung volumes to a greater extent. Furthermore, the impact of position was greater in the OHS group suggesting that EFL may be a contributing factor in the development of hypercapnic respiratory failure in these individuals.

\section{S57 AEROBIC TRAINING AND DETRAINING IN COPD AND HEALTHY CONTROLS}

B Popat ${ }^{1}$, L Latimer ${ }^{1}$, L Houchen-Wolloff', C Bolton ${ }^{2},{ }^{1} \mathrm{M}$ Steiner. ${ }^{1}$ NIHR Respiratory BRU, Glenfield Hospital, Leicester, UK; ${ }^{2}$ Nottingham Respiratory Research Unit, Nottingham, UK

10.1136/thoraxjnl-2014-206260.63

Introduction Deconditioning is a key cause of exercise intolerance in COPD patients. Exercise training provides an effective method of improving aerobic exercise performance in this group. There is poor understanding on the trajectory of changes

Abstract S56 Table 1 Differences in expiratory flow limitation, as demonstrated by $\Delta \mathrm{X}_{\mathrm{rS}}$ between healthy controls, OSA and OHS

\begin{tabular}{|c|c|c|c|c|c|c|c|c|c|}
\hline & \multicolumn{3}{|l|}{ Healthy controls } & \multicolumn{3}{|c|}{ Obstructive Sleep Apnoea } & \multicolumn{3}{|c|}{ Obesity Hypoventilation Syndrome } \\
\hline & Upright Seated & $45^{\circ}$ & Supine & Upright Seated & $45^{\circ}$ & Supine & Upright Seated & $45^{\circ}$ & Supine \\
\hline $\mathrm{DX}_{\mathrm{rs}, 5 \mathrm{~Hz}}\left(\mathrm{cmH}_{2}\right.$ O.s. $\left.\mathrm{L}^{-1}\right)$ & $-0.15 \pm 0.13$ & $0.04 \pm 0.21^{*}$ & $-0.03 \pm 0.20$ & $0.52 \pm 0.74$ & $1.18 \pm 1.51$ & $2.05 \pm 2.23$ & $2.33 \pm 2.96$ & $4.45 \pm 2.91$ & $5.18 \pm 2.65^{*}$ \\
\hline$\%$ of EFL breaths & $0 \pm 0$ & $0 \pm 0$ & $0 \pm 0$ & $0 \pm 0$ & $20 \pm 27$ & $38 \pm 3$ & $35 \pm 45$ & $68 \pm 4$ & $73 \pm 34^{*}$ \\
\hline \multicolumn{10}{|l|}{ Inspiratory Reactance } \\
\hline$X_{\text {insp }, 5 \mathrm{~Hz}}\left(\mathrm{cmH}_{2}\right.$ O.s. $\left.\mathrm{L}^{-1}\right)$ & $-0.55 \pm 0.21$ & $-0.67 \pm 0.43$ & $-0.69 \pm 0.43$ & $-0.93 \pm 0.89$ & $-2.47 \pm 1.66$ & $-1.91 \pm 0.91^{*}$ & $-2.60 \pm 0.95$ & $-3.38 \pm 1.80$ & $-2.69 \pm 1.75$ \\
\hline \multicolumn{10}{|l|}{ Inspiratory Resistance } \\
\hline $\mathrm{R}_{\text {insp, } 5 \mathrm{~Hz}}\left(\mathrm{cmH}_{2}\right.$ O.s. $\left.\mathrm{L}^{-1}\right)$ & $2.71 \pm 0.55$ & $3.45 \pm 0.66$ & $3.96 \pm 0.99^{*}$ & $4.31 \pm 1.34$ & $6.20 \pm 1.35$ & $6.43 \pm 1.52^{*}$ & $5.61 \pm 1.86$ & $6.42 \pm 1.05$ & $7.46 \pm 1.11^{*}$ \\
\hline R5-19insp & $-0.23 \pm 0.18$ & $0.04 \pm 0.21$ & $0.19 \pm 0.31^{*}$ & $0.37 \pm 0.49$ & $1.29 \pm 0.96$ & $1.29 \pm 0.56^{*}$ & $1.91 \pm 0.89$ & $2.20 \pm 0.60$ & $2.21 \pm 0.81$ \\
\hline Ti/Ttot & $0.44 \pm 0.04$ & $0.45 \pm 0.05$ & $0.45 \pm 0.03$ & $0.43 \pm 0.04$ & $0.45 \pm 0.03$ & $0.45 \pm 0.04$ & $0.41 \pm 0.05$ & $0.41 \pm 0.07$ & $0.43 \pm 0.07$ \\
\hline VE (L/min) & $14.26 \pm 7.06$ & $13.5 \pm 5.19$ & $14.62 \pm 7.58$ & $15.17 \pm 5.67$ & $17.72 \pm 5.02$ & $15.83 \pm 4.75$ & $20.88 \pm 9.44$ & $13.71 \pm 3.32$ & $13.3 \pm 2.62$ \\
\hline RR (bpm) & $15.75 \pm 1.99$ & $17.9 \pm 2.93^{*}$ & $17.5 \pm 2.95^{*}$ & $20.61 \pm 7.48$ & $21.59 \pm 6.52$ & $21.7 \pm 5.46$ & $22.14 \pm 6.90$ & $20.84 \pm 3.79$ & 19.321 .71 \\
\hline
\end{tabular}


made in exercise performance between COPD and Healthy controls (HC), during aerobic training and more importantly limited data exists on the rates of detraining between the groups.

Methods This study measures sub-maximal and maximal performance in response to an aerobic training programme and to a period of detraining.

COPD and HC undertook 8 weeks of supervised cycling exercise training three times a week. There consequently followed a 4 week period of detraining, and resumption of pre cycling habitual activity (not engaging in regular exercise). A symptom limited incremental cycle (ramp protocol) and constant work rate (submaximal/endurance) cardiopulmonary exercise tests (CPET) were performed at baseline, after 4 and 8 weeks of training and after detraining. Cycling training intensity and CPET endurance work were equivalent to $65 \%$ of the Work (in Watts (W)) at VO2 Peak during the baseline CPET ramp test. Training intensity was re-set if there was any improvement during the 4-week CPET ramp test. Results 10 COPD patients (MRC 3, 2 males, age 74 years, FEV 1 $63.5 \%$ predicted) and 7 HC (MRC 1, 4 males, age 71 years, FEV $_{1}$ $111 \%$ predicted) completed the study. COPD group had lower starting training workloads (59.5 vs 121 Watts, $\mathrm{p}<0.05$ ) compared to HC.

HC showed a significant increase in Peak VO2 uptake in the ramp but COPD patients only showed an increasing trend. There were however increases in the time achieved during sub-maximal testing in both groups during the 8 week training period. However during detraining, there was relative preservation in the $\mathrm{HC}$ but a significant reduction in endurance time in the COPD group.

(All values median, unless stated)

Conclusion/discussion Exercising training at moderate intensities showed no changes in maximal performance in COPD groups, compared to HC. However gains in sub-maximal performance were seen in both groups. Training induced gains in sub-maximal performance may be better preserved in HC during detraining, when compared to the COPD groups.

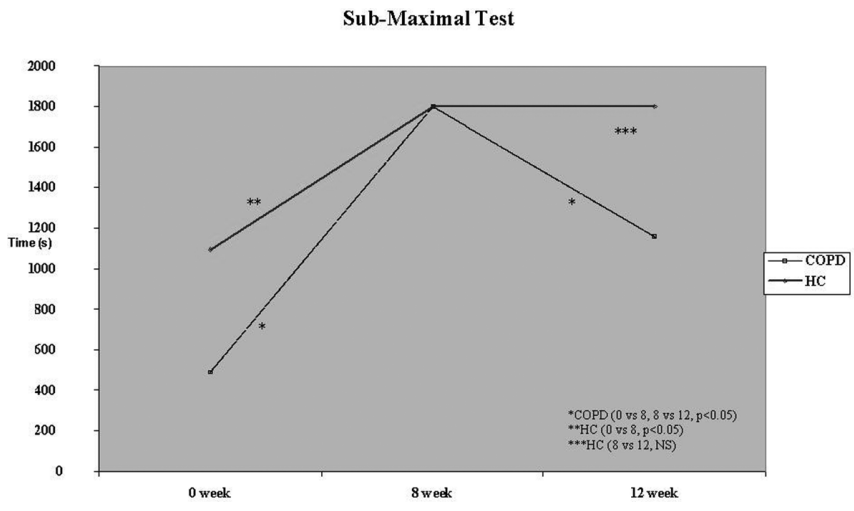

Abstract S57 Figure 1 Sub-Maximal Test

\section{Latent TB and biomarkers}

\section{S58 PROSPECTIVE HEALTH ECONOMIC EVALUATION OF DIFFERENT RECOMMENDED STRATEGIES FOR TB TESTING IN A CONTEMPORARY HIV POSITIVE COHORT}

${ }^{1} \mathrm{SJ}$ Capocci, ${ }^{2} \mathrm{~S}$ Sewell, ${ }^{2} \mathrm{C}$ Smith, ${ }^{1} \mathrm{I}$ Cropley, ${ }^{1} \mathrm{~S}$ Bhagani, ${ }^{2} \mathrm{~S}$ Morris, ${ }^{1} \mathrm{M}$ Johnson, ${ }^{1} \mathrm{MCl}$ Lipman. 'Royal Free London NHS Foundation Trust, London, UK; ${ }^{2}$ University College London, London, UK

\subsection{6/thoraxjnl-2014-206260.64}

Introduction The risk of active tuberculosis (TB) disease is estimated to be increased 40-fold in people with HIV (PHIV).
Effective antiretroviral treatment (ART) may reduce this significantly. UK national guidance recommends using blood interferon gamma release assay (IGRA) +/- tuberculin skin testing (TST) for latent TB (LTBI) diagnosis but there are little supporting health economic data. We sought to evaluate the cost-effectiveness of different testing strategies using data from a prospective contemporary cohort.

Methods Subjects receiving ambulatory HIV care were recruited by stratified selection within our HIV centre. TST, IGRA (TSpot. TB), frontal chest radiograph (CXR) and single induced sputum for mycobacterial culture were performed. The yield was used to model a screening programme that utilised current UK HIV demographics (Public Health England). Costs were based on the BNF, local costs or published literature (TST $£ 16$, IGRA $£ 60$, CXR $£ 50$, induced sputum $£ 42$, treatment for latent and active TB $£ 786$ and $£ 7619$ respectively). Uptake and LTBI treatment efficacy were both estimated at $65 \%$. We assumed a lifetime reactivation rate with TST+/IGRA + of $10 \%$ and TST+/IGRA- of $2 \%$; and that all those with evidence of LTBI would be given treatment.

Results Over 13 months, 211 people were recruited. 26\% were female and 26\% black African. LTBI rates amongst subjects from sub-Saharan Africa, medium and low TB incidence countries were $8 / 55$ (15\%), 2/31 (6\%) and 4/125 (3\%) respectively. One patient had a persistently indeterminate IGRA. Subclinical TB disease was diagnosed in two (1\%) subjects.

Using these data to model TB testing nationally, the British HIV Association (BHIVA) testing algorithm was the most costeffective with an incremental cost effectiveness ratio (ICER) of $£ 21,475$. The NICE algorithm both cost more and prevented fewer cases of active TB (Table 1). More comprehensive strategies were associated with increasing cost.

Conclusion Testing only those at highest risk of progression to active TB disease in an HIV population with high ART use was cost-effective, whilst most strategies testing all comers and for active TB cost considerably more than the $£ 20-30,000 /$ QALY gained threshold used in the UK.

\section{S59 EVALUATING THE CLINICAL UTILITY OF XPERT® MTB/ RIF FOR THE DIAGNOSIS AND MANAGEMENT OF TUBERCULOSIS IN A HIGH BURDEN REGION OF THE UK}

J Kim, B O'Connor, H Patel, N Perera, M Wiselka, G Woltmann, P Haldar. University of Leicester, Leicester, UK

\subsection{6/thoraxjnl-2014-206260.65}

Introduction The importance of Xpert ${ }^{\circledR}$ MTB/RIF (GeneXpert) for the rapid confirmation of tuberculosis (TB) and indicator for drug resistance is now well established for high burden, resource poor countries, with a high prevalence of smear positive pulmonary disease. The phenotype of disease differs significantly in high income countries like the UK with fewer smear positive cases and more extra-pulmonary disease. The role of GX in this setting is unclear.

Objectives To evaluate how GeneXpert was being used in local practice and determine factors that influenced test performance. Methods We performed a retrospective analysis of all GeneXpert tested patient samples between October 2011 and April 2014. Out of area sample requests and unprocessed specimens were excluded. Positive GeneXpert results were categorised as very low, low, medium and high. Sensitivity and specificity analyses excluded clinically diagnosed TB without supporting evidence and stratified samples by type and smear status. Statistical analyses were computed on SPSS (v.16). 